\title{
Ab initio View of Emergent Symplectic Symmetry and Its Crutial Role in Nuclear Dynamics
}

\author{
T. Dytrych ${ }^{1,2}$, K.D. Launey ${ }^{2}$, J.P. Draayer ${ }^{2}$, D. Langr ${ }^{3}$ \\ ${ }^{1}$ Nuclear Physics Institute of the Czech Academy of Sciences, \\ 25068 Řež, Czech Republic \\ ${ }^{2}$ Department of Physics and Astronomy, Louisiana State University, \\ Baton Rouge, LA 70803, USA \\ ${ }^{3}$ Department of Computer Systems, Faculty of Information Technology, \\ Czech Technical University in Prague, 16000 Praha, Czech Republic
}

Received 5 November 2021

doi: https://doi.org/10.55318/bgjp.2022.49.1.037

\begin{abstract}
Results of large-scale first principle nuclear structure studies using the symmetry-adapted no-core shell model are reported. It is shown that nuclei up through the intermediate-mass region display highly regular and ubiquitous patterns of dominant nuclear shapes that vibrate and rotate. This emergent structure is tied to an approximate symplectic $\operatorname{Sp}(3, \mathbb{R})$ symmetry, and it is shown to determine dominant features of low-lying states, even in close-to-spherical nuclear states without any recognizable rotational properties.
\end{abstract}

KEY WORDS: Ab initio nuclear theory, nuclear collective motion, $\operatorname{Sp}(3, \mathbb{R})$ group, $\mathrm{U}(3)$ group, symmetry-adapted basis.

\section{Introduction}

The ab initio nuclear many-body approaches represent a long-sought unifying framework for describing and understanding nuclear phenomena as it emerges from the microscopic degrees of freedom tied to the underlying principles and symmetries of QCD. The challenge of understanding the emergence of nuclear collective modes from ab initio considerations stems from the combinatorial increase in the problem size with the number of nucleons and model space cutoff. When expressed in terms of literally billions of Slater determinants, the structure of nuclear wave functions becomes quickly unrecognizable. This problem can be addressed by utilizing a many-nucleon basis that reflects symmetries inherent to the studied system. In atomic nuclei such symmetries were recognized early on by Elliott [1] followed by work of Rowe and Rosensteel [2], who identified 


\section{T. Dytrych et al.}

the symplectic group $\operatorname{Sp}(3, \mathbb{R})$ and its subgroup $U(3)$ as the underlying symmetries of microscopic nuclear collective motion. Aiming to understand dominant modes of nuclear collective dynamics emerging from ab initio studies, we constructed the symmetry-adapted no-core shell model [3-5] (SA-NCSM) approach that solves the nuclear many-body problem with realistic nucleon-nucleon interactions using many-nucleon basis organized in terms of $\mathrm{U}(3)$ irreducible representations (irreps).

In the present investigation, we unveil the crucial role of the symplectic symmetry in large-scale ab initio SA-NCSM results for various nuclei and nuclear realistic interactions. We first describe U(3) symmetry-adapted basis of SA-NCSM, followed by an overview of $\operatorname{Sp}(3, \mathbb{R}) \supset \mathrm{U}(3)$ symplectic shell model basis. Next, we present a method for expanding symplectic basis states in terms of $\mathrm{U}(3)$ basis of the SA-NCSM. Finally, we present results that illustrate a remarkable outcome from first-principles investigations up through the intermediate-mass region, namely, the simplicity of nuclear low-lying states and the striking dominance of the symplectic $\operatorname{Sp}(3, \mathbb{R})$ symmetry of nuclear collective dynamics.

\section{$2 \mathrm{U}(3)$ Symmetry-Adapted Basis}

The SA-NCSM adopts powerful mathematical algorithms of group theory [6, 7] to decompose a given model space in terms of $\mathrm{U}(3)$ irreps constructed in proton-neutron formalism with the spatial part of basis classified by the $\mathrm{SU}(3) \supset$ $\mathrm{SO}$ (3) group-subgroup chain [8]. Thereby each many-nucleon basis state can be schematically labeled as

$$
\left|\left\{\begin{array}{c}
\vec{\alpha}_{p} N_{p}\left(\lambda_{p} \mu_{p}\right) \\
\vec{\alpha}_{n} N_{n}\left(\lambda_{n} \mu_{n}\right)
\end{array}\right\} N \rho(\lambda \mu) \kappa L ;\left(S_{p} S_{n}\right) S ; J M\right\rangle .
$$

Here, $N_{p}$ and $N_{n}$ denote the total number of harmonic oscillator (HO) excitation quanta for protons and neutrons, $N=N_{p}+N_{n}$. Protons and neutrons are organized into $\mathrm{SU}(3)$ irreps labeled by $\left(\lambda_{p} \mu_{p}\right)$ and $\left(\lambda_{n} \mu_{n}\right)$ quantum numbers. Proton and neutron irreps are coupled to proton-neutron SU(3) irrep $(\lambda \mu)$. The symbol $\rho$ denotes a multiplicity label which is needed to distinguish between multiple occurrences of the $(\lambda \mu)$ irrep within the product of coupling. The $\mathrm{SU}(3)$ quantum numbers are related to the quadrupole deformation of a nucleus. For example, $(00),(\lambda 0)$, and $(0 \mu)$, describe spherical, prolate, and oblate deformation, respectively. The quantum numbers $\left(S_{p} S_{n}\right) S$ denote the total intrinsic spin of protons and neutrons coupled to the total intrinsic spin of a nucleus. The total spin $S$ is coupled with the total orbital angular momentum $L$ to the total angular momentum $J$ and its projection $M$. The multiplicity label $\kappa$ reflects the fact that multiple occurrences of $L$ are possible within a general irrep $(\lambda \mu)$ of $\mathrm{SU}(3)$. The symbol $\vec{\alpha}_{p}\left(\vec{\alpha}_{n}\right)$ schematically denotes all the additional quantum numbers needed to uniquely determine proton and neutron irreps, see, e.g., ref [8] for details. 


\section{Emergent Symplectic Symmetry}

\section{Symplectic $\operatorname{Sp}(3, \mathbb{R})$ Symmetry-Adapted Basis}

The symplectic model [2] was proposed by Rosensteel and Rowe as a microscopic model of nuclear collective phenomena that includes monopole and quadrupole collective vibrations as well as vorticity degrees of freedom for a description of rotational dynamics in a continuous range from irrotational to rigid rotor flows. It was derived as a microscopic generalization of the successful Bohr-Mottelson-Frankfurt collective model. The symplectic model can be regarded as a many HO shell extension od the Elliott's SU(3) model and hence it allows one to realize nuclear collective states in terms of the shell model configurations.

The relevance of the symplectic $\operatorname{Sp}(3, \mathbb{R})$ symmetry for a description of a system of strongly interacting nucleons emerges from the physical relevance of its 21 generators. The important operators such as, e.g., many-nucleon HO, the manyparticle kinetic energy, the mass monopole and quadrupole moments, angular momentum operators, are elements of the symplectic algebraic structure.

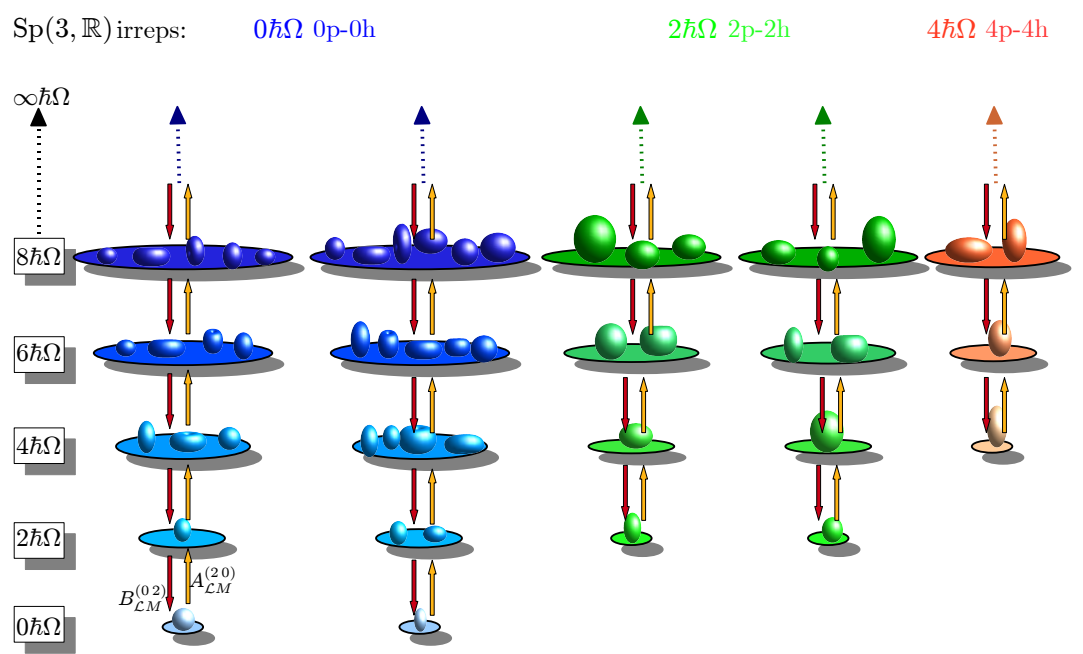

Figure 1. (Color online) The schematic plot illustrating decomposition of the shell model space in terms of the symplectic $\operatorname{Sp}(3, \mathbb{R})$ irreps. Each $\operatorname{Sp}(3, \mathbb{R})$ irrep is depicted as a cone made up of $\mathrm{U}(3)$ irreps, which are shown as ellipsoids. Each ellipsoid thus represents $\operatorname{Sp}(3, \mathbb{R}) \supset \mathrm{U}(3)$ basis states labeled by $N(\lambda \mu)$ quantum numbers that are related to a definite nuclear deformation. The action of the symplectic raising and lowering operators $\hat{A}^{(20)}$ and $\hat{B}^{(02)}$ within a $\operatorname{Sp}(3, \mathbb{R})$ irrep is also schematically depicted. Figure adapted from [9]. 


\section{T. Dytrych et al.}

The symplectic shell model group-subgroup chain, $\operatorname{Sp}(3, \mathbb{R}) \supset \mathrm{U}(3) \supset \mathrm{SO}(3)$, is directly responsible for the computational tractability of the symplectic model. It transcends the Elliott $\mathrm{SU}(3)$ group and as a consequence bridges between the microscopic shell model and the nuclear collective dynamics. The shell model structure of the $\operatorname{Sp}(3, \mathbb{R})$ generators is elucidated if they are realized as

$$
\begin{aligned}
& \hat{A}_{i j}=\sum_{n=1}^{A} b_{n i}^{\dagger} b_{n j}^{\dagger} \\
& \hat{B}_{i j}=\sum_{n=1}^{A} b_{n i} b_{n j} \\
& \hat{C}_{i j}=\frac{1}{2} \sum_{n=1}^{A}\left(b_{n i}^{\dagger} b_{n j}+b_{n j} b_{n i}^{\dagger}\right),
\end{aligned}
$$

where $b_{n i}^{\dagger}$ and $b_{n i}$ are the HO raising and lowering operators for the $n$th particle and $i, j=\mathrm{x}, \mathrm{y}$, or $\mathrm{z}$. The operators $C_{i j}$, generators of $\mathrm{U}(3)$ group, act only within a major $\mathrm{HO}$ shell, while the six operators $A_{i j}$ are $2 \hbar \Omega$ raising operators and their adjoint $B_{i j}$ are $2 \hbar \Omega$ lowering operators.

Symplectic basis states that span a given $\operatorname{Sp}(3, \mathbb{R})$ irrep are build by the action of the symplectic raising operators $\hat{A}_{i j}$ on a single $\mathrm{U}(3)$ irrep, dubbed symplectic bandhead. Each symplectic bandhead carry a definite number of HO quanta $N_{0}$ and can be associated through its $\mathrm{SU}(3)$ quantum numbers $\left(\lambda_{0} \mu_{0}\right)$ to a certain equilibrium shape. The action of symmetrically coupled polynomials in $\hat{A}_{i j}$ on the symplectic bandhead generates monopole and quadrupole vibrations and rotations of the equilibrium shape. The resulting symplectic basis states are labeled as

$$
\left|\alpha_{0} N_{0}\left(\lambda_{0} \mu_{0}\right) N_{n}\left(\lambda_{n} \mu_{n}\right) N \rho(\lambda \mu) \kappa L ;\left(S_{p} S_{n}\right) S ; J M\right\rangle,
$$

where the multiplicity index $\alpha_{0}$ is generally needed to distinguish between multiple equivalent symplectic bandheads. Quantum numbers $N_{n}\left(\lambda_{n} \mu_{n}\right)$ provide a $\mathrm{U}(3)$ tensorial character of a polynomial in $\hat{A}_{i j}$ that acts on the bandhead. Finally, $\mathrm{U}(3)$ set of quantum numbers $N(\lambda \mu)$ labels the symplectic state, where $N=N_{0}+N_{n}$ denotes the total number of HO quanta. In the proton-neutron formalism, each symplectic irrep carries the total intrinsic spin of protons, neutron, and the total spin, $S_{p}, S_{n}$, and $S$.

The problem of evaluating a realistic Hamiltonian in the symplectic basis is challenging. It is hindered by the fact that $\operatorname{Sp}(3, \mathbb{R})$ Clebsch-Gordan coefficients are not available. This problem can be avoided by the method built on ideas of Suzuki and Hecht [10,11], who derived recursive formulas for computation of matrix elements of a generic $\mathrm{U}(3)$ unit tensor operator. This method was recently implemented and applied in the study of emergence of collectivity in ab initio results for ${ }^{7} \mathrm{Be}[12]$. 


\section{Emergent Symplectic Symmetry}

An alternative method is build on the fact that each $\operatorname{Sp}(3, \mathbb{R})$ basis state (5) can be expanded in terms of U(3) symmetry-adapted states of SA-NCSM (1) that carry identical $\left(S_{p} S_{n}\right) S ; N(\lambda \mu)$ quantum numbers. Such a space of equivalent $\mathrm{U}(3)$ irreps constitutes a mere fraction of $N \hbar \Omega$ shell model subspace of the Hilbert space. Moreover, once the expansion is known, one can readily utilize the existing SA-NCSM computational framework to carry out nuclear structure calculations in $\operatorname{Sp}(3, \mathbb{R})$ symmetry-adapted basis.

\section{Expanding Symplectic States in U(3) Symmetry-Adapted Basis}

The expansion of symplectic $\operatorname{Sp}(3, \mathbb{R}) \supset \mathrm{U}(3)$ states in terms of $\mathrm{U}(3)$ symmetryadapted basis of the SA-NCSM framework can be obtained by solving the eigenvalue problem for the second order $\operatorname{Sp}(3, \mathbb{R})$ Casimir operator,

$$
\hat{C}_{2}^{\mathrm{Sp}(3, \mathbb{R})}=\left[\hat{A}^{(20)} \times \hat{B}^{(02)}\right]^{(00)} .
$$

Each $\operatorname{Sp}(3, \mathbb{R})$ basis state is an eigenstate of the operator $\hat{C}_{2}^{\mathrm{Sp}(3, \mathbb{R})}$ with the associated eigenvalue

$$
C_{2}^{\mathrm{Sp}(3, \mathbb{R})}\left(\lambda_{0}, \mu_{0}, N_{0}, \lambda, \mu, N\right)=\frac{1}{2 \sqrt{6}}\left[C(\lambda, \mu, N)-C\left(\lambda_{0}, \mu_{0}, N_{0}\right)\right] .
$$

Here

$$
C(\lambda, \mu, N)=\frac{2}{3}\left(\lambda^{2}+\mu^{2}+\lambda \mu+3 \lambda+3 \mu\right)+\frac{1}{3} N^{2}-4 N,
$$

where $N_{0}$ and $N$ are related to the eigenvalue of the intrinsic $A$-particle threedimensional isotropic $\mathrm{HO}$, i.e.

$$
N=\sum_{i}^{A}\left(\eta_{i}+\frac{3}{2}\right)-\frac{3}{2} .
$$

As the Casimir operator is both $\mathrm{U}(3)$ and intrinsic spin scalar, the eigenvalue problem can be solved in a subspace of equivalent $\mathrm{U}(3)$ irreps, that is irreps that carry the same $\left(S_{p} S_{n}\right) S ; N(\lambda \mu)$ quantum numbers. Furthermore, resulting expansion coefficients do not depend on downstream quantum numbers $k, L$, and $J$. Indeed, utilizing $\mathrm{U}(3)$ symmetry-adapted basis leads to a substantial reduction in the problem size.

In our construction method, the symplectic generators $\hat{A}_{i j}$ and $\hat{B}_{i j}$ are not translationally invariant. In order to obtain $\operatorname{Sp}(3, \mathbb{R})$ states with the center-of-mass in the $\mathrm{HO}$ ground state, we augment the Casimir operator (6) with the Lawson term $\hat{N}_{c m}$ represented by the operator counting the number of center-of-mass HO quanta, which is multiplied by a sufficiently large strength parameter $\lambda_{c m}$. 


\section{T. Dytrych et al.}

In addition, the operator $\hat{C}_{2}^{\mathrm{Sp}(3, \mathbb{R})}$ is multiplied by a negative sign to ensure that $\mathrm{Sp}(3, \mathbb{R})$ states with the center-of-mass in the $\mathrm{HO}$ ground state are associated with negative eigenvalues. Therefore, we are solving the eigenvalue problem for the lowest lying eigenstates of operator

$$
\hat{O}_{\lambda_{c m}} \equiv-\hat{C}_{2}^{\mathrm{Sp}(3, \mathbb{R})}+\lambda_{c m} \hat{N}_{c m}
$$

The spectrum of the operator $\hat{O}_{\lambda_{c m}}$, which we denote $\sigma\left(\hat{O}_{\lambda_{c m}}\right)$, consists of the three regions:

1. If $\sigma\left(\hat{O}_{\lambda_{c m}}\right)<0 \Longrightarrow$ eigenstates are $\operatorname{Sp}(3, \mathbb{R})$ states with the centerof-mass in the $\mathrm{HO}$ ground state.

2. If $\sigma\left(\hat{O}_{\lambda_{c m}}\right)=0 \Longrightarrow$ eigenstates are $\operatorname{Sp}(3, \mathbb{R})$ bandheads with the center-of-mass in the $\mathrm{HO}$ ground state.

3. If $\sigma\left(\hat{O}_{\lambda_{c m}}\right) \geq \min \left(-C_{2}^{\mathrm{Sp}(3, \mathbb{R})}\right)+\lambda_{c m} \Longrightarrow$ eigenstates are either $\operatorname{Sp}(3, \mathbb{R})$ states or $\operatorname{Sp}(3, \mathbb{R})$ bandheads with the excited center-of-mass contribution.

The resulting eigenvectors realize $\operatorname{Sp}(3, \mathbb{R})$ symmetry-adapted basis states and can be readily used to construct the Hamiltonian in the new symplectic basis. While this procedure is computationally intensive, especially for higher- $N$ sets of large dimensionality, the expansion needs to be carried out only once for a given nucleus. Furthermore, the Hamiltonian matrix in $\operatorname{Sp}(3, \mathbb{R})$ basis of most dominant symplectic irreps is drastically small in size and the nuclear energies and states can be calculated without the need for supercomputers.

\section{Results}

We generated the complete $\mathrm{Sp}(3, \mathbb{R})$ symmetry-adapted basis with up to $12 \mathrm{HO}$ excitation quanta (i.e. model space including $14 \mathrm{HO}$ major shells) of the oddodd ${ }^{6} \mathrm{Li}$ and even-even ${ }^{8} \mathrm{He}$ nuclei. For the intermediate-mass nuclei ${ }^{20} \mathrm{Ne}$, we constructed symplectic basis spanning a physically relevant subspace that includes configurations with up to 8 excited $\mathrm{HO}$ quanta (i.e. including $11 \mathrm{HO}$ major shells). For our study, we use ab initio SA-NCSM wave functions obtained with the Entem-Machleidt (EM) N3LO [13] and NNLOopt [14] chiral potentials without renormalization in nuclear medium.

The projection of SA-NCSM wave functions onto $\operatorname{Sp}(3, \mathbb{R})$ basis reveals that low-lying nuclear states, deformed or not, are predominantly (70\%-80\%) composed of a few $\operatorname{Sp}(3, \mathbb{R})$ irreps as depicted in Figure 2. This implies that nuclei exhibit relatively simple physics. Nuclear collective dymamics as it emerges from first principle nuclear structure studies can be desribed by a few equilibrium shapes that vibrate and rotate, where each equilibrium shape is associated with a single symplectic $\operatorname{Sp}(3, \mathbb{R})$ irrep.

For example, the ground state and its rotational band in ${ }^{6} \mathrm{Li}$ and ${ }^{20} \mathrm{Ne}$ are dominated from $70-80 \%$ by a single $\mathrm{Sp}(3, \mathbb{R})$ irrep built over the most deformed 


\section{Emergent Symplectic Symmetry}

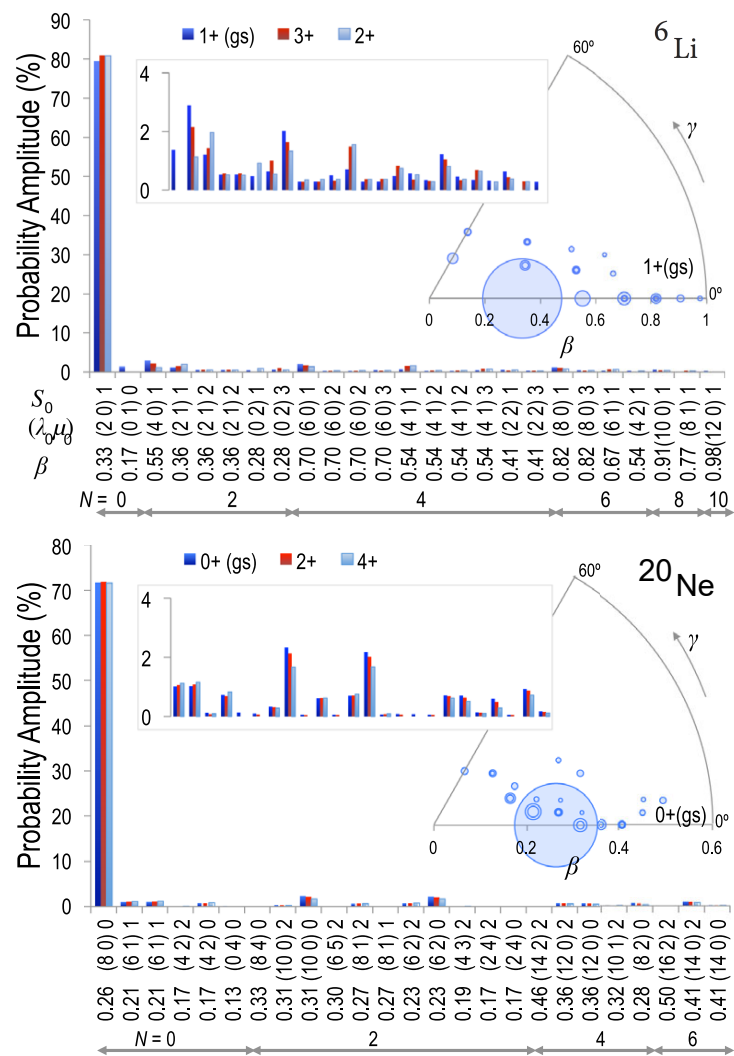

Figure 2. (Color online) Projection of ab initio SA-NCSM ground states and their rotational bands in ${ }^{6} \mathrm{Li}$ (top) and ${ }^{20} \mathrm{Ne}$ (bottom) onto $\mathrm{Sp}(3, \mathbb{R})$ irreps labeled by the deformation parameter $\beta, \mathrm{SU}(3)$ labels $\left(\lambda_{0} \mu_{0}\right)$, and the total intrinsic spin $S$. The insets show the same irreps excluding the most dominant ones, together with the $\beta-\gamma$ plot for the ground state. The SA-NCSM wave functions were obtained using the EM-N3LO interaction in a model space of $14\left({ }^{6} \mathrm{Li}\right)$ and $11\left({ }^{20} \mathrm{Ne}\right) \mathrm{HO}$ major shells, and $\hbar \Omega=20 \mathrm{MeV}$. Figure adapted from [5].

$\mathrm{U}(3)$ valence space bandhead, i.e. $\left(\lambda_{0} \mu_{0}\right)=(20)$ in the case of ${ }^{6} \mathrm{Li}$, and $\left(\lambda_{0} \mu_{0}\right)=(80)$ in the case of ${ }^{20} \mathrm{Ne}$. It is remarkable that for both nuclei the model space defined by this single dominant $\operatorname{Sp}(3, \mathbb{R})$ irrep closely reproduces experimental excitation energies as well as $B(E 2)$ strengths (Figure 3 ). This can be understood from the fact that $E 2$ transitions operator is a $\operatorname{Sp}(3, \mathbb{R})$ generator that can not mix different symplectic irreps. This implies that the largest fraction of these transitions, and hence nuclear collectivity, necessarily emerges within this most dominant symplectic irrep. It is important to note that this holds true also for the point-particle root-mean-square matter radii, as $r^{2}$ operator is also a $\operatorname{Sp}(3, \mathbb{R})$ generator [15]. 
T. Dytrych et al.

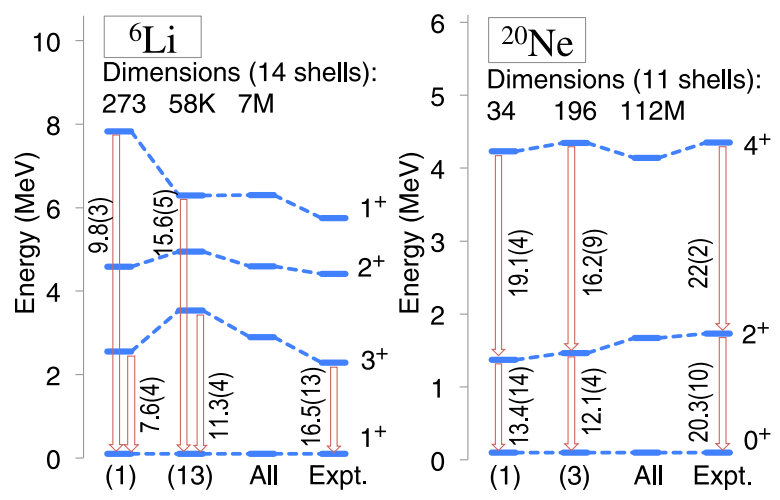

Figure 3. (Color online) Excitation energies and $B(E 2)$ transition strenghts (in W.u.) for ${ }^{6} \mathrm{Li}$ and ${ }^{20} \mathrm{Ne}$ calculated in the ab initio SA-NCSM with $\operatorname{Sp}(3, \mathbb{R})$ basis using only a small number (specified in the $\mathrm{x}$-axis labels) of dominant symplectic irreps including the most dominant one, as compared to experiment ("Expt."). Model-space dimensions are shown above each case; for comparison, the corresponding complete model space dimension for $J^{\pi}=0^{+}, 2^{+}, 4^{+}$in ${ }^{20} \mathrm{Ne}$ in $11 \mathrm{HO}$ shells is $3.8 \times 10^{10}$. Figure adapted from [5].

The observed dominance of $\operatorname{Sp}(3, \mathbb{R})$ symmetry extends to low-lying states of studied nuclei. We identified low-lying states that have almost identical $\operatorname{Sp}(3, \mathbb{R})$ structure to that of the ground state, but which differ in their orbital angular momenta quantum number $L$. This is illustrated in Figure 2 for ${ }^{6} \mathrm{Li}$ and ${ }^{20} \mathrm{Ne}$. Such a coherent structure is a rigorous signature of rotations of a shape and can be used to identify members of a rotational band and enhanced $B(E 2)$ strengths.

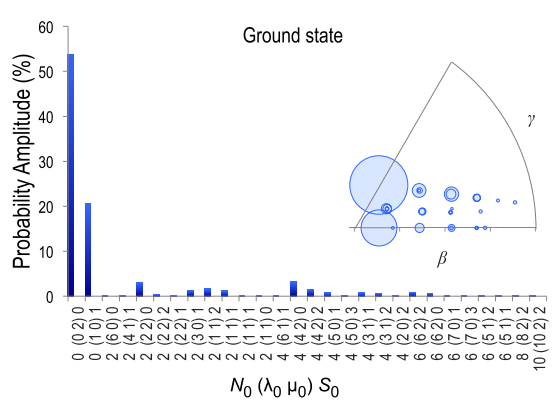

(a)

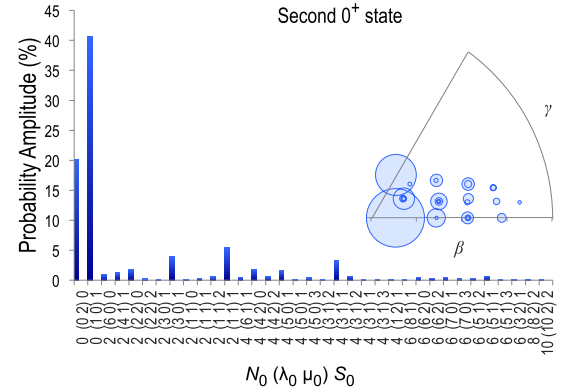

(b)

Figure 4. (Color online) Projection of SA-NCSM wavefunctions for (a) the $0^{+}$ground state and (b) the second $0^{+}$state of ${ }^{8} \mathrm{He}$ onto $\mathrm{Sp}(3, \mathbb{R})$ irreps labeled by the total $\mathrm{HO}$ excitations $N_{0}, \mathrm{SU}(3)$ labels $\left(\lambda_{0} \mu_{0}\right)$, and the total intrinsic spin $S$, together with the corresponding $\beta-\gamma$ plot. The wave funtions were obtained using the NNLOopt interaction in a complete model space of $14 \mathrm{HO}$ major shells and $\hbar \Omega=20 \mathrm{MeV}$. Adapted from [15]. 


\section{Emergent Symplectic Symmetry}

We also studied the two lowest $0^{+}$states in ${ }^{8} \mathrm{He}$, which has been suggested to be a halo nucleus. Although ${ }^{8} \mathrm{He}$ is considered to be spherical, both studied states are dominated by the prolate $(10)$ and the oblate $(02) \operatorname{Sp}(3, \mathbb{R})$ irreps. The dominant irrep contributes at the 40-55\% level and a secondary in importance contributes approximately by $20 \%$ (Figure 4). Clearly, the structure of both states can be described as an interplay of two shapes, which on average may appear to have a zero deformation, but with a $B(E 2)$ strength from its $2^{+}$rotational state that constructively adds the nonzero contributions of both shapes [15].

\section{Conclusions}

We presented a method for the construction of symplectic $\operatorname{Sp}(3, \mathbb{R})$ symmetryadapted basis states in terms of $\mathrm{U}(3)$ symmetry-adapted basis states of the SANCSM. This method allows us to study symplectic $\operatorname{Sp}(3, \mathbb{R})$ symmetry of nuclear collective motion as it emerges in large-scale ab initio nuclear structure results, while addressing the long-standing problem of carrying out ab initio nuclear structure calculations in the symplectic basis that typically represents a mere fraction of a complete model space. Outcomes point to unexpectedly ubiquitous symplectic symmetry, with the illustrative examples provided for ${ }^{6} \mathrm{Li}$, ${ }^{8} \mathrm{He}$, and ${ }^{20} \mathrm{Ne}$ nuclei. We find that ground states and low-lying excited states are predominantly comprised (typically in excess of 70\%-80\%) of only a few $\operatorname{Sp}(3, \mathbb{R})$ irreps. We showed that these dominant irreps constitute physically relevant model spaces of small dimension that holds key to describing fundamental collective features observed in atomic nuclei. In short, our results expose the role of the $\operatorname{Sp}(3, \mathbb{R})$ symmetry as a remarkably good symmetry of the strong nuclear force in the low-energy regime.

\section{Acknowledgements}

The work was supported by the U.S. National Science Foundation (OIA1738287, ACI-1713690, PHY-1913728), SURA, and in part by the Czech Ministry of Education, Youth and Sports under Grant No. CZ.02.1.01/0.0/0.0/16_019/0000765. Financial support by the Bulgarian National Science Fund under the contract number KP-06-N28/6 is gratefully acknowledged.

\section{References}

[1] J.P. Elliott (1958) Collective motion in the nuclear shell model I. Classification schemes for states of mixed configurations. Proc. Roy. Soc. A 245128.

[2] G. Rosensteel, D.J. Rowe (1977) Nuclear Sp(3, R) model. Phys. Rev. Lett. 3810.

[3] T. Dytrych et al. (2013) Collective modes in light nuclei from first principles. Phys. Rev. Lett. 111252501

[4] K.D. Launey, T. Dytrych, J.P. Draayer (2016) Symmetry-guided large-scale shellmodel theory. Prog. Part. Nucl. Phys. 89101. 
T. Dytrych et al.

[5] T. Dytrych et al. (2020) Physics of Nuclei: Key Role of an Emergent Symmetry. Phys. Rev. Lett. 124042501.

[6] J.P. Draayer, Y. Leschber, S.C. Park, R. Lopez (1989) Representations of U(3) in U(n). Comp. Phys. Comm. 56279.

[7] D. Langr, T. Dytrych, J.P. Draayer, K.D. Launey, P. Tvrdík (2019) Efficient algorithm for representations of U(3) in U(N). Comp. Phys. Comm. 244442.

[8] D. Langr, T. Dytrych, J.P. Draayer, K.D. Launey (2019) Accelerating many-nucleon basis generation for high performance computing enabled ab initio nuclear structure studies. Int. J. High Perform. Comput. Appl. 33(3) 522.

[9] T. Dytrych, K.D. Sviratcheva, J.P. Draayer, C. Bahri, J.P. Vary (2008) Ab initio symplectic no-core shell model. J. Phys. G: Nucl. Part. Phys. 35123101.

[10] Y. Suzuki, K.T. Hecht (1986) Symplectic and cluster excitations in nuclei:: Evaluation of interaction matrix elements. Nucl. Phys. A 455315.

[11] Y. Suzuki (1986) Cluster and symplectic excitations in nuclei. Nucl. Phys. A 448 395.

[12] A.E. McCoy, M.A. Caprio, T. Dytrych, P.J. Fassano (2020) Emergent Sp(3,R) dynamical symmetry in the nuclear many-body system from an ab initio description. Phys. Rev. Lett. 125102505.

[13] E. Epelbaum (2006) Few-nucleon forces and systems in chiral effective field theory. Prog. Part. Nucl. Phys. 57654.

[14] A. Ekström et. al (2013) Optimized chiral nucleon-nucleon interaction at next-tonext-to-leading order. Phys. Rev. Lett. 110192502.

[15] K.D. Launey, T. Dytrych, G.H. Sargsyan, R.B. Baker, J.P. Draayer (2020) Emergent symplectic symmetry in atomic nuclei: Ab initio symmetry-adapted no-core shell model. Eur. Phys. J. Sp. Top. 2292429. 